\title{
Optical spectroscopy of functionalized gold nanoparticles assemblies as a function of the surface coverage
}

\author{
C. Humbert • O. Pluchery • E. Lacaze • A. Tadjeddine • \\ B. Busson
}

Published online: 24 November 2013

(C) The Author(s) 2013. This article is published with open access at SpringerLink.com

\begin{abstract}
Layers of thiophenol functionalized spherical gold nanoparticles grafted on $\mathrm{Si}(100)$ are probed by linear UV-vis, Fourier transform infrared and nonlinear infrared-visible vibrational sum/difference-frequency generation spectroscopies as a function of the nanoparticles surface coverage. Depending on the dipping time (5 $\mathrm{min}, 20 \mathrm{~min}, 1 \mathrm{~h}$, and $24 \mathrm{~h}$ ) in the colloidal solution, AFM imaging corroborates that the silicon surface coverage with gold nanoparticles increases, while the distance between neighbouring nanoparticles decreases, leading to their aggregation which dramatically impacts their optical properties. In the UV-vis reflectance spectra after the appearance of the $525-\mathrm{nm}$ individual plasmonic band, a second broad band located at $660 \mathrm{~nm}$ and related to the gold nanoparticles aggregation on silicon rapidly dominates in intensity. Nonlinear vibrational spectroscopy is able to detect the specific vibration of the thiophenol molecules (3, $055 \mathrm{~cm}^{-1}$ ) whatever the immersion time and at least down to $1 \%$ of the substrate filling factor by the gold nanoparticles, overtaking the molecular sensitivity threshold of surface infrared and Raman spectroscopies on small gold nanostructures $(17 \mathrm{~nm})$ adsorbed on a semiconductor. Moreover, a quantitative analysis of the nonlinear vibrational fingerprint from $5 \mathrm{~min}$ to $24 \mathrm{~h}$ in the framework of the effective medium models of Maxwell-Garnett and Bruggeman illustrates the role played by the interband and the plasmonic properties of gold modulated by the silicon optical response. In this case, the sample reflectivity affects the molecular oscillator strength
\end{abstract}

Electronic supplementary material The online version of this article (doi:10.1007/s13404-013-0126-5) contains supplementary material, which is available to authorized users.

C. Humbert $(\bowtie) \cdot$ A. Tadjeddine $\cdot$ B. Busson

Univ Paris-Sud, Laboratoire de Chimie Physique, CNRS,

Bâtiment 201 Porte 2, 91405 Orsay, France

e-mail: christophe.humbert@u-psud.fr

O. Pluchery $\cdot$ E. Lacaze

Univ Pierre et Marie Curie UPMC-CNRS, Institut des Nanosciences de Paris, 4 place Jussieu, 75005 Paris, France measured by nonlinear optical vibrational spectroscopy. For this latter technique, no coupling with the optical properties of aggregated AuNps is evidenced while the localized surface plasmon resonance excitation amplifies the molecular response.

Keywords Gold · Nanospheres · Silicon · Nonlinear optics · UV-visible spectroscopy $\cdot$ Atomic force microscopy

\section{Introduction}

The manufacturing of well-designed nanosensors is a key step to improve their sensitivity threshold in the probe-target scheme encountered in molecular recognition, especially when expecting the detection of materials traces to the single molecule level. Among the numerous methods of sensors design at the nanoscale, metal-based supports are routinely used taking advantage of their plasmonic properties [1]. In fact, their interest lies in the enhancement due to the surface plasmon resonance (SPR) of the nano-objects organized in networks with well-defined optical properties in the UVvisible (UV-vis) spectral range, depending on the nanoobject size, shape and metal nature and on the lattice parameter. All these properties are related to the surface preparation. A silicon substrate is generally used as a transducer to take benefit of the particular optical and electronic properties of those systems. Nowadays, different ways of production are routinely used. Among them, one route consists in a physical method based on controlled deposition directly on the substrate by electron beam lithography [2]. Another well-known route is "wet" chemistry where metal nanoparticles are synthesized first, then grafted on the substrate through aminosilane molecular interlayer [3]. While the latter method seems easier to implement, its difficulty lies in preventing the aggregation of the nano-objects on the substrate and in their subsequent functionalization. These steps are crucial because 
it should not quench or shift significantly the SPR intensity or position, respectively, with respect to the SPR properties observed in the native colloidal solution. Indeed, spherical gold nanoparticles (AuNps) are commonly used to build nanosensors with a fixed SPR located in the visible light.

Nanosensors are based on a molecular recognition process evidenced by monitoring the SPR shift. For an efficient use of these sensors, a perfect knowledge of the chemistry of the process at play is mandatory, but plasmonics alone does not enlighten which molecular bonds are involved in the recognition of the target molecule by the probe molecule. To reach that goal, vibrational optical techniques are preferred such as infrared and Raman spectroscopic tools. PM-IRRAS and SERS are efficient for providing molecular recognition at the nanoscale level. Nevertheless, on silicon, the selection rules of PM-IRRAS prevent enhanced sensitivity. In SERS, the unequalled sensitivity can reach the single molecule level but is directly related to the presence of "hot spots", generally occurring on samples where nanoparticles are touching, which requires preparation of specific samples. An alternative method, whose sensitivity could be independent of the sample preparation, consists in taking advantage of the IR and Raman peculiar rules together: nonlinear optical sum (difference)frequency generation spectroscopy (SFG/DFG) based on the mixing of two incident visible and infrared beams at the same point of the probed interface. This latter generates a SFG/DFG coherent light beam depending on the modulation of the afforded incident energy by IR molecular absorption and/or visible SPR enhancement (Fig. 1). Therefore, the main advantage of this technique for nanotechnological devices

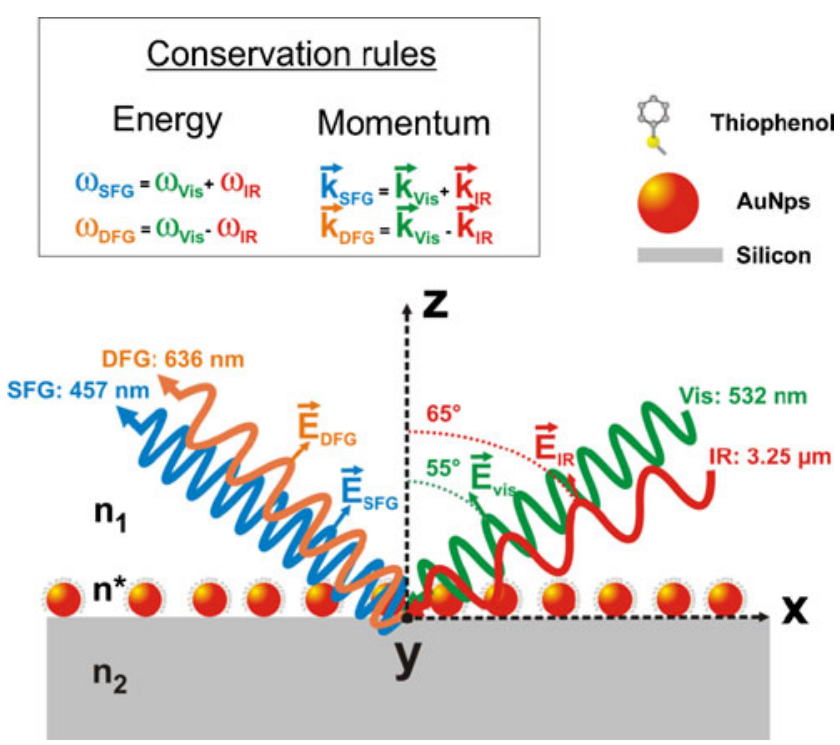

Fig. 1 Sketch of the SFG and DFG processes on the thiophenol/AuNps/ $\mathrm{Si}(100)$ interface. All the beams are p-polarised, i.e. the wavevectors $\mathrm{k}$ are located in the $X Z$ plane. The incident infrared and visible laser beams are always mixed at the same point of the probed interface whether the SFG or DFG configuration characterization lies in its interface intrinsic molecular sensitivity at the sub-monolayer level and its potential coupling to the SPR resonance as recently demonstrated by considering samples based on solid substrate as platforms for nanosensors, an amplification factor of the molecular SFG signal was put in evidence for functionalized AuNps films with respect to a flat gold reference surface. AuNps were deposited either on silicon in external reflection configuration [4] or on glass substrates in total internal reflection configuration [5] to compare the sensitivity and molecular ordering of functionalized AuNp films as a function of the surface coverage on glass [6], to unravel the orientation of grafted molecules on AuNps and AgNps [7] and to extract and deduce the nature of the vibration modes thanks to the SPR amplification on functionalized AuNp films in the fingerprint spectral range of aromatic molecules with density functional theory (DFT) calculations [8]. In a general way, SFG has also proven its efficiency in biomolecular recognition on glass substrate [9], in DNAbased biosensors on Pt(111) single crystal [10] and in enlightening DNA hybridization on glass [11] and (100)-facetted gold films [12]. In summary, SFG/DFG spectroscopy is a promising tool in describing the chemistry occurring in biological recognition $[13,14]$ performed on nanostructured biosensors provided that we light on/off the SPR amplification in a controlled manner: AuNps nature, size, shape, and dispersion (surface coverage and lattice parameter) on the substrate, exciting visible wavelength. In those SFG/DFG works, no systematic analysis of the effect of an evolving plasmonic pattern on the level of the molecular sensitivity threshold, i.e. sub-monolayer sensitivity was achieved.

Therefore, we address in this paper the role of the plasmonic pattern on the sensitivity threshold for different nanostructured interfaces, as a function of the surface coverage by varying only one parameter: the surface coverage with AuNps on a silicon substrate that was previously functionalized with aminosilane monolayer. This AuNps coverage is controlled by the immersion time of the sample in colloidal AuNps suspension. The surface optical and chemical properties are therefore studied by grafting thiophenol molecules on the AuNps as a probe of the SPR enhancement and/or coupling to the vibrational activity and as a specific marker of the particle surface. To reach that goal, a careful pre-characterization step is necessary: atomic force microscope (AFM) imaging to deduce the surface coverage properties (AuNps diameter and dispersion on the silicon), UV-vis spectroscopy in reflection on the silicon substrate to obtain the plasmonic shape. Fourier transform infrared spectroscopy (FTIR) is used as a standard technique to give the chemical fingerprint of the sample and defined its sub-monolayer sensitivity threshold. Finally, SFG/DFG spectroscopy is performed to give the vibrational pattern of the interface in order to deduce the potential SPR coupling effect and its efficiency to improve the molecular sensitivity at the nanoscale. 


\section{Experimental details}

Sample preparation

AuNps were synthesized according to the Turkevich method where $1 \mathrm{~mL}$ of $8.5 \times 10^{-4} \mathrm{M}$ trisodium citrate $\left(\mathrm{Na}_{3} \mathrm{C}_{6} \mathrm{H}_{5} \mathrm{O}_{7}\right)$ was added to a boiling aqueous (Millipore water, resistivity= $18 \mathrm{MOhm} \mathrm{cm})$ solution of $20 \mathrm{~mL} \mathrm{HAuCl}_{4}\left(2.5 \times 10^{-4} \mathrm{M}\right)$ under vigorous agitation. The resulting solution $(\mathrm{pH}=5.5)$ displays a UV-vis absorbance spectrum (see Fig. 5) with a single peak located at $520 \mathrm{~nm}[4,15]$ corresponding to non-aggregated AuNps with diameter $=17 \pm 2 \mathrm{~nm}$. Wafers of ultrasonically cleaned $\mathrm{n}$-doped silicon $\left(1 \times 1 \mathrm{~cm}^{2}\right.$, Siltronix) were silanized in an absolute methanol solution containing 3-aminopropyl-triethoxysilane (APTES, $\mathrm{H}_{2} \mathrm{~N}\left(\mathrm{CH}_{2}\right)_{3} \mathrm{Si}\left(\mathrm{OC}_{2} \mathrm{H}_{5}\right)_{3}, 10 \%$ vol.). The surface deposition of the four samples was achieved by dipping each silanized wafer in an equal amount of the native colloidal aqueous solution during $5 \mathrm{~min}, 20 \mathrm{~min}, 1 \mathrm{~h}$ and $24 \mathrm{~h}$, respectively. No particle aggregation in the solutions was observed during and after the dipping as checked by UV-vis spectroscopy. After the AuNps grafting, samples were functionalized during $18 \mathrm{~h}$ in a $10^{-3} \mathrm{M}$ thiophenol solution $\left(\mathrm{C}_{6} \mathrm{H}_{5} \mathrm{SH}\right)$ dissolved in dichloromethane $\left(\mathrm{CH}_{2} \mathrm{Cl}_{2}\right)$. All chemicals were purchased from Sigma-Aldrich.

\section{AFM microscopy}

We make use of AFM imaging (Digital Instrument, DI3100) in tapping mode to analyze the surface density of the samples. The silicon tips have $130 \mathrm{KHz}$ working frequency, with a curvature radius at the apex around $10 \mathrm{~nm}$. It does not allow sufficient lateral resolution to image correctly isolated gold nanoparticles, but it is sufficient to identify each nanoparticle and to obtain their diameter, equal to their height with respect to the substrate: $17 \pm 2 \mathrm{~nm}$.

\section{FTIR spectroscopy}

FTIR measurements have been carried out to identify the vibrational modes of thiophenol in the range 2,500-3, $400 \mathrm{~cm}^{-1}$. In this range, silicon is transparent for the infrared beam and the measurement is performed in transmission geometry at $60^{\circ}$ of incidence with a Bruker Tensor- 27 spectrometer. The absorbance $\mathrm{A}=-\log \left(I / I_{0}\right)$ is calculated by recording $I_{0}$ from the sample itself before thiophenol was deposited. In order to maximize the sensitivity, FTIR spectra were recorded by averaging over 1,000 scans with a spectral resolution of $4 \mathrm{~cm}^{-1}$. The noise was as low as $3 \times 10^{-5}$ absorbance units so that it was possible to identify surface species at sub-monolayer coverage.
Nonlinear optical vibrational spectroscopy

The sum/difference-frequency generation spectroscopic setup is described elsewhere [6]. Briefly, it is based on a 15 ps pulsed laser Nd:YAG source $(1 \mu \mathrm{s}$ train, repetition rate $25 \mathrm{~Hz}$ ). After amplification, one part is used to pump an infrared optical parametric oscillator (OPO) built around a $\mathrm{LiNBO}_{3}$ crystal giving access to the $2,500-4,000 \mathrm{~cm}^{-1}$ spectral range (10 $\mu \mathrm{J}$ pulse energy, $3 \mathrm{~cm}^{-1} \mathrm{OPO}$ bandwidth). The other part is used to obtain a green visible laser beam $(5 \mu \mathrm{J}$ pulse energy and $532 \mathrm{~nm}$ wavelength) by frequency-doubling in a BBO crystal. The infrared and visible beams are then mixed at the same point of the probed surface for each sample with angles of incidence of $65^{\circ}$ and $55^{\circ}$ with respect to the surface normal, respectively. The infrared, visible and SFG/DFG beams are p-polarized. All the SFG/DFG data are normalized to the SFG/DFG signal of a $\mathrm{ZnSe}$ reference crystal in order to compensate for eventual laser fluctuations or atmospheric absorption. The geometrical configuration depicted in Fig. 1 is the same for each sample. The only difference resides in the detection scheme for SFG and DFG. In fact, SFG and DFG signals have a different emission direction. In these conditions, to switch from SFG to DFG configuration, only one mirror is tilted in the lateral direction to send either SFG or DFG photons in the same direction for their detection after spatial and spectral filtering through a monochromator. To compensate for eventual misalignment of the SFG detection direction, the baseline of the SFG spectra is recovered by a linear fit of the experimental data. In order to compare quantitatively the SFG data with respect to the DFG data, an experimental scaling factor set to 1.55 has to be applied to the DFG vibration mode amplitudes reported in Tables 1 and 2 of "SFG/DFG measurements". It is easily explained by considering Fig. 1 because of the nonuniform response characteristics over the visible wavelength range of the detection chain (gratings and photomultipliers) for SFG and DFG energies. The SFG photons are detected in the blue while the DFG photons are detected in the red.

Within this experimental configuration, SFG and DFG spectroscopies respect the rules of the energy ( $\hbar \omega)$ and momentum $(\mathrm{k})$ conservation parallel to the interface (i.e. the processes are phase-matched). Both spectroscopies sum up two contributions: one coherent (phase-matched SFG/DFG) and one scattered [16]. Sum-frequency scattering contribution has been measured from the bulk colloidal solutions of bigger particles $[16,17]$. Nevertheless, it is negligible in our experiments due to the small size of the particles and to the very small thickness of the monolayer. In addition, the scattering angle distribution completely differs from the phase-matching angle, and we have checked experimentally that the SFG/DFG 
Table 1 Evolution of the AuNps surface density $\mathrm{N}_{\mathrm{s}}$, filling factor $f$ and fitting parameters of the SFG/DFG spectra

\begin{tabular}{|c|c|c|c|c|c|c|c|c|}
\hline \multirow{2}{*}{$\begin{array}{l}\text { Optical probe } \\
\text { Dipping time }\end{array}$} & \multicolumn{4}{|l|}{ SFG } & \multicolumn{4}{|l|}{ DFG } \\
\hline & $5 \min$ & $20 \mathrm{~min}$ & $1 \mathrm{~h}$ & $24 \mathrm{~h}$ & $5 \min$ & $20 \mathrm{~min}$ & $1 \mathrm{~h}$ & $24 \mathrm{~h}$ \\
\hline $\mathrm{N}_{\mathrm{S}}\left(10^{10} / \mathrm{cm}^{2}\right)$ & $1.05 \pm 0.12$ & $2.48 \pm 0.48$ & $6.2 \pm 1.28$ & $9.48 \pm 0.96$ & $1.05 \pm 0.12$ & $2.48 \pm 0.48$ & $6.2 \pm 1.28$ & $9.48 \pm 0.96$ \\
\hline$f(\%)$ & $1.57 \pm 0.17$ & $3.80 \pm 0.72$ & $9.18 \pm 1.93$ & $14.95 \pm 1.49$ & $1.57 \pm 0.17$ & $3.80 \pm 0.72$ & $9.18 \pm 1.93$ & $14.95 \pm 1.49$ \\
\hline$\left|\mathrm{C}_{\mathrm{FG}}\right|$ & $0.074 \pm 0.008$ & $0.096 \pm 0.019$ & $0.098 \pm 0.02$ & $0.11 \pm 0.011$ & $0.023 \pm 2.5 \mathrm{e}^{-4}$ & $0.054 \pm 0.011$ & $0.098 \pm 0.021$ & $0.005 \pm 5 \mathrm{e}^{-4}$ \\
\hline$\phi_{\mathrm{FG}}\left(^{\circ}\right)$ & 91.61 & 88.5 & 57.49 & 85.05 & 26.67 & 18.45 & -17.61 & 23.78 \\
\hline$\left|a_{0}\right|$ & $0.076 \pm 0.008$ & $0.14 \pm 0.028$ & $0.53 \pm 0.111$ & $0.64 \pm 0.064$ & $0.11 \pm 0.012$ & $0.23 \pm 0.046$ & $0.56 \pm 0.118$ & $0.48 \pm 0.062$ \\
\hline$\omega_{0}\left(\mathrm{~cm}^{-1}\right)$ & $3,056.59$ & $3,049.76$ & $3,057.95$ & $3,050.58$ & $3,060.8$ & $3,058.17$ & $3,055.56$ & $3,053.94$ \\
\hline
\end{tabular}

signals shown in this paper propagate along the phasematched direction.

\section{UV-visible spectroscopy}

UV-visible (UV-vis) experiments are carried out to obtain the optical signature of the AuNps monolayer deposited on the silicon substrate. In the UV-vis range, this signature is mostly influenced by the plasmon resonance of the nanoparticles. Since the silicon substrate is a reflecting material in the UVvis range, the spectra were recorded in reflection geometry with an incidence angle of $10^{\circ}$ and with a Cary 5 spectrophotometer (Varian). However, in order to discriminate the optical signature of the particles from the typical reflectivity spectrum of bare silicon, a differential method is performed. The reflectivity $R_{0}$ of silicon with APTES is recorded and used as a reference. After the reflectivity $R$ of the sample with AuNps is measured, the reflectance is processed: Reflectance $=-\log (R /$ $R_{0}$ ). It requires a precise alignment of the reference and the sample within the same measurement procedure in order to avoid spectral features related to different optical paths. In these conditions, we checked that the measurement reproducibility is ensured at a level better than $1 \times 10^{-3}$ absorbance units.

\section{Results and discussion}

\section{AFM measurements}

We show the representative and typical AFM pictures of our four samples differing by the AuNps surface coverage (monitored by the dipping time in the colloidal solution) in Fig. 2 (from left to right: $5 \mathrm{~min}-20 \mathrm{~min}-1 \mathrm{~h}-24 \mathrm{~h}$ ). In order to ensure the accuracy of the AFM measurements and have a correct count of the AuNps to deduce their surface density $\left(\mathrm{N}_{\mathrm{s}}\right)$, multiple scans are performed at different scales $\left(10 \times 10 \mu \mathrm{m}^{2}-1 \times 1 \mu \mathrm{m}^{2}-500 \times 500 \mathrm{~nm}^{2}\right)$ on several areas of the silicon wafers. As shown on Fig. 2, each individual nanoparticle can be identified which allows to obtain from the AFM images of $1 \mu^{2}$ and $500 \times 500 \mathrm{~nm}^{2}$ the local surfacic density of gold nanoparticles. For each sample, we have averaged on at least five different areas on the sample, the density variation for each sample being indicated in Table 1. From these measurements, in addition to the AuNps height (i.e. the diameter), we can deduce that the AuNps surface density $\left(\mathrm{N}_{\mathrm{s}}\right)$ reported in Table 1 ("SFG/DFG measurements") is multiplied by a factor of $\sim 10$ when increasing the dipping time from $5 \mathrm{~min}$ to $24 \mathrm{~h}$. It is worth noting that it does not evolve significantly on the timescale between 1 and $24 \mathrm{~h}$. From $\mathrm{N}_{\mathrm{s}}$ and by considering gold spheres ( $\sim 17 \mathrm{~nm}$ diameter), we can deduce the volumic filling factor $f$ of the gold inclusions grafted on the silicon substrate with respect to an equivalent surface of bare silicon. Moreover, by performing profile measurement in the lateral direction, it is possible to evaluate the average distance between the centres of two AuNps. We observe that they can be in close contact for $24 \mathrm{~h}$ dipping time $\left(\mathrm{d}_{\mathrm{np}-\mathrm{np}} \sim 20 \mathrm{~nm}\right)$. In these conditions, it is expected that the optical properties will be drastically modified as checked by UV-visible measurements.

\section{FTIR measurements}

The differential spectra acquired with FTIR spectroscopy are aimed at detecting when the thiophenol vibrational bands in the 2,500-3,400 $\mathrm{cm}^{-1}$ can be detected. Figure 3 clearly shows the $\mathrm{CH}$ stretching vibration modes of the thiophenol aromatic core at $3,057 \mathrm{~cm}^{-1}$ from $1 \mathrm{~h}$ immersion time. We checked that when a sample without AuNps is dipped into the thiophenol solution, no band is detected at $3,057 \mathrm{~cm}^{-1}$. This demonstrates that thiophenol exclusively interacts with gold. Therefore, the stretching mode is detected for $f>9 \%$. Nevertheless, in the case of a very low amount of AuNps on the surface such as $f$ between 1 and $4 \%$, FTIR spectroscopy is not sensitive enough. Moreover, the IR peak is fairly broad which does not allow identifying clearly all these features. It should be noted that the correct assignment of this vibration mode requires being careful because it is a rich spectral range influenced by the molecular adsorption on gold atoms [18]. Whatever the precise nature of the vibration mode, it should have a sufficient infrared activity to be detected in FTIR. 
Moreover, from the SFG/DFG principles, it should also have simultaneously an important Raman activity to be active. The interested reader will find in the Electronic Supplementary Information some discussion based on literature and DFT calculations on this peculiar point.

\section{SFG/DFG measurements}

We present the corresponding SFG and DFG measurements of the four samples in Fig. 4 left and right, respectively. In both cases, we observe the presence of a dip (SFG) or a peak (DFG) at the frequency $\omega_{0}=3,055 \pm 6 \mathrm{~cm}^{-1}$ interfering with a strong (SFG baseline) or weak (DFG baseline) background depending on the immersion time in the colloidal solution. This vibrational feature corresponds to the stretching vibration mode of the $\mathrm{CH}$ groups of the aromatic core of the thiophenol [4] that we already mentioned in the FTIR measurements. Whatever $f$, we always observe the vibration mode from 1 to $15 \%$ contrary to FTIR spectroscopy which is a first proof of an exaltation of the molecular signal due to the gold LSPR.

For both nonlinear configurations, we observe that the vibration mode intensity increases with the immersion time in the AuNps solution, which is evidently related to the increasing $\mathrm{N}_{\mathrm{s}}$ as deduced from AFM measurements. We want to know how much SFG/DFG could be quantitative and how it is possible to assess the quantity of adsorbed thiophenol on the AuNps. To that end, the SFG/DFG intensity in reflection mode (coherent emission) is modelled by the following equation [8]:

$\mathrm{I}_{\mathrm{FG}}\left(\omega_{\mathrm{IR}}\right)=\left|\chi_{\mathrm{eff}}^{(2)}\right|^{2}=\left|\mathrm{C}_{\mathrm{FG}} \mathrm{e}^{\mathrm{i} \phi_{\mathrm{FG}}}+\frac{\mathrm{a}_{0}}{\omega_{\mathrm{IR}}-\omega_{0} \pm \mathrm{i} \Gamma_{0}}\right|^{2}$.

We then apply the SFG/DFG data fitting developed in a procedure detailed elsewhere [8]. Moreover, to allow a direct comparison of $\mathrm{a}_{0}$ as a function of the dipping time, the bandwidth of the vibration mode ( $\Gamma_{0}$ damping constant $)$ is set to $7.5 \mathrm{~cm}^{-1}$ in the equation of the intensity $\mathrm{I}_{\mathrm{FG}}$ where the subscript FG stands either for SFG $\left(+\mathrm{i} \Gamma_{0}\right)$ or DFG $\left(-\mathrm{i} \Gamma_{0}\right) \cdot \chi_{\mathrm{eff}}^{(2)}$ is the nonlinear second order effective susceptibility of the interface, which includes the surface reflectivity (Fresnel factors). In order to obtain a more quantitative description of the molecular vibration, we thus need to correct $\mathrm{a}_{0}$ using Fresnel factors, leading to the so-called absolute molecular amplitude $\mathrm{a}_{\mathrm{zzz}} \cdot \mathrm{C}_{\mathrm{FG}}$ and $\phi_{\mathrm{FG}}$ are the amplitude and phase of the nonresonant (NR) contribution to the nonlinear signals. The evolution of the relevant parameters as a function of $\mathrm{N}_{\mathrm{s}}$ obtained from the fitting procedure is given in Table 1. In our case, the NR contribution, which appears as a strong (SFG) or weak (DFG) baseline, depends on the AuNps electronic properties and more precisely to $\mathrm{s}-\mathrm{d}$ interband electronic transition of gold as well-established for single crystals and colloidal 


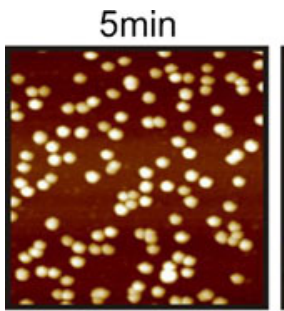

$$
\begin{gathered}
f=1.57 \% \\
d_{n p-n p}=66 n m
\end{gathered}
$$

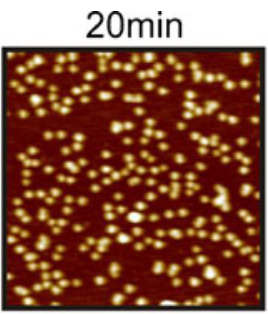

$$
\begin{gathered}
f=3.80 \% \\
d_{n p-n p}=48 n m
\end{gathered}
$$

Fig. 2 AFM pictures (width scale: $1 \times 1 \mu \mathrm{m}^{2}$ for $5 \mathrm{~min}$ to $1 \mathrm{~h}, 500 \times$ $500 \mathrm{~nm}^{2}$ for $24 \mathrm{~h}$; height scale: $30 \mathrm{~nm}$ from black to white) of the four thiophenol/AuNps/APTES/Si(100) interfaces as a function of the

materials $[4,8]$. This point will be discussed further in "Influence of the interband transition character of gold nanospheres on the SFG/DFG spectra".

We equally observe a discrepancy of the $\phi_{\mathrm{FG}}$ parameter for the sample immersed $1 \mathrm{~h}$ in the AuNps solution when looking at the SFG/DFG spectra. A different interference pattern is observed. We have no definitive explanation for that effect. It suggests that another chemical component may interact with the thiophenol (a strong $\mathrm{OH}$ band due to the presence of water molecules) for this specific preparation conditions.

In our experimental configuration, a direct comparison between the different $\mathrm{a}_{\mathrm{o}}$ as a function of $\mathrm{N}_{\mathrm{s}}$ makes sense provided that the surface reflectivity is taken into account via the Fresnel factors $\left(\mathrm{F}_{\mathrm{zzz}}\right)$ contribution to the SFG/DFG signals. In fact, as the polarization scheme of the SFG/DFG experiment is ppp (for SFG/DFG, visible and IR beams, respectively) as depicted in Fig. 1, only the $\mathrm{F}_{\mathrm{zzz}}$ contribution is significant [8]. To address this point, we consider a three-

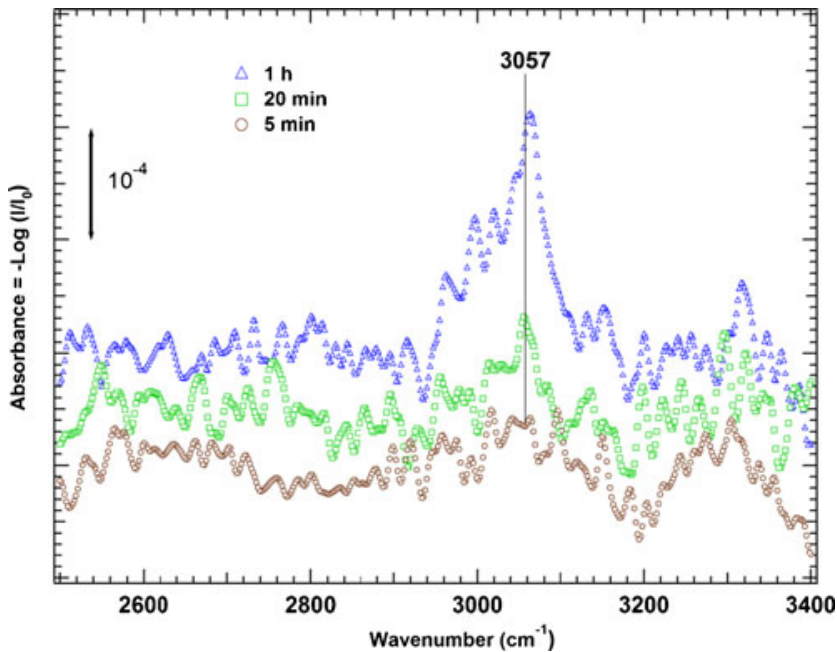

Fig. 3 FTIR differential spectra showing the $\mathrm{CH}$ stretching mode of thiophenol adsorbed on the AuNps. The reference spectrum corresponds to the same samples but before adsorption of thiophenol. No clear thiophenol vibration can be detected on the spectra taken with a low amount of AuNps (5-20 min dipping time). With a greater amount of AuNps ( $1 \mathrm{~h}$ dipping time), a broad vibration mode clearly emerges from the noise
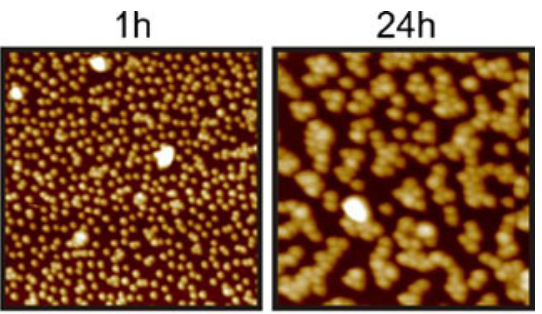

$$
\begin{array}{cc}
f=9.18 \% & f=14.95 \% \\
\mathrm{~d}_{n p-n p}=33 n m & \mathrm{~d}_{n p-n p}<30 \mathrm{~nm}
\end{array}
$$

immersion time in the colloidal solution. Relevant parameters are equally given: filling factor $(f)$ and AuNps separation $\left(\mathrm{d}_{\mathrm{np}-\mathrm{np}}\right)$

layer model, $\mathrm{N}_{\mathrm{s}}$ being therefore included in an effective medium model [19] where the wavelengths of light (450-4, $000 \mathrm{~nm})$ are much greater than the surface roughness $(\sim 17 \mathrm{~nm})$. The appropriate effective medium model has to be selected depending on the filling factor $f$ describing the gold inclusions in a host matrix. If we develop two extreme cases, the Maxwell-Garnett (MG) model for low $f(1-10 \%)$ and the Bruggeman (BM) model for high $f(>30 \%)$, we can deduce and compare the absolute molecular amplitude $\left(\mathrm{a}_{\mathrm{zzz}}\right)$

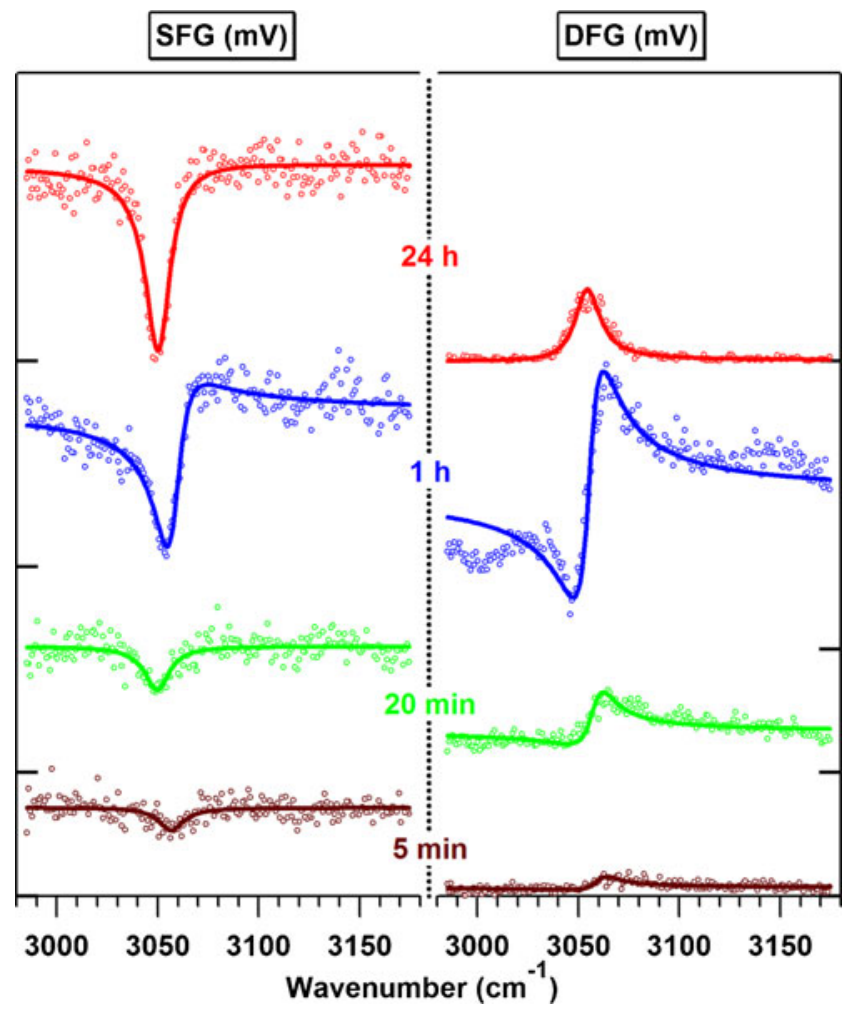

Fig. 4 SFG (left panel) and DFG (right panel) spectra of the four thiophenol/AuNps/APTES/Si(100) interfaces as a function of the increasing immersion time in the colloidal solution ( $5 \mathrm{~min}-20 \mathrm{~min}-1 \mathrm{~h}-24 \mathrm{~h}$ ). SFG and DFG intensity scales are identical ( $\mathrm{min}, 0 \mathrm{mV}$; max, $18 \mathrm{mV}$ ) to facilitate direct comparison between SFG and DFG data whatever the immersion time. The zero of each curve is indicated by dashes on the left (SFG) and right (DFG) axes 
of the SFG and DFG signals for the four samples as reported in Table 2. In this manner, we will be able to investigate the possible importance of the sample reflectivity on the results.

The difference between MG and BM lies in the role attributed to the dielectric constant $\varepsilon^{*}$ of the composite layer of refractive index $n^{*}$ located between the silicon substrate $\left(n_{2}\right)$ and the ambient air $\left(n_{1}\right)$ in the three-layer model as depicted in Fig. 1. In the MG model, the composite layer is defined as a host matrix of air $\left(\varepsilon_{\mathrm{h}}=1\right)$ with a low concentration of gold spherical inclusions. In the BM model, because of the high concentration of gold inclusions, the matrix host is considered as the composite layer $\left(\varepsilon_{\mathrm{h}}=\varepsilon^{*}\right)$, i.e. each of its component is considered as an inclusion. The interested reader will find in the Electronic Supplementary Material the details of the procedure used to deduce $\mathrm{a}_{\mathrm{zzz}}$ from the SFG and DFG data in the framework of both effective medium models as well as the Fresnel factors calculations. We finally find, as discussed further on Fig. 6, that the vibrational amplitude is essentially proportional to the actual molecular coverage $\mathrm{N}_{\mathrm{s}}$ for both SFG/DFG measurements when considering the appropriate effective medium model.

\section{UV-vis measurements}

We present the corresponding UV-vis reflectance curves of the four samples in Fig. 5. The optical features are strongly modified with the immersion time in the colloidal solution. The localized surface plasmon resonance (LSPR) of AuNps is expected between 505 and $520 \mathrm{~nm}$ depending on the immediate molecular surrounding of the particles. The absorbance of AuNps exhibits a positive peak localized at $520 \mathrm{~nm}$ in solution and at $515 \mathrm{~nm}$ when deposited on glass and measured

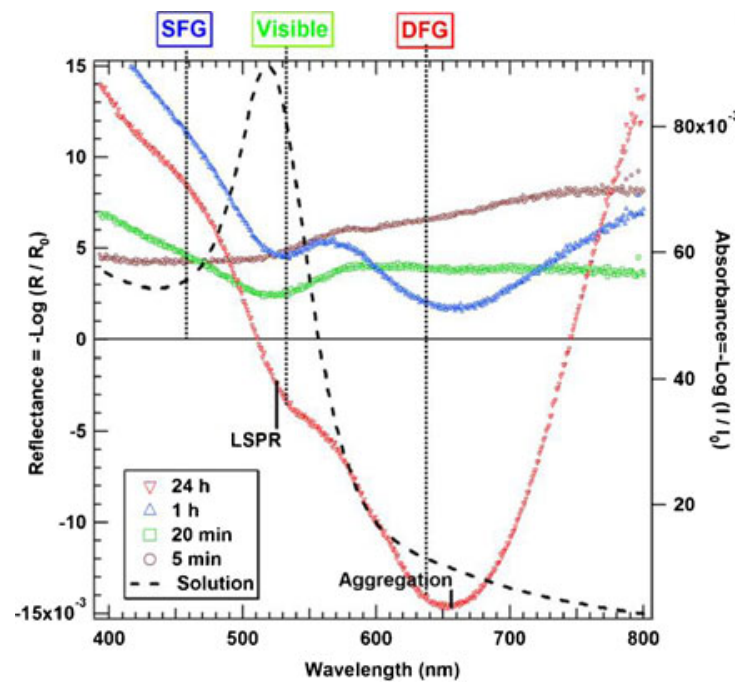

Fig. 5 Left: UV-vis absorbance curve of the AuNps native colloidal solution and reflectance curves of the four thiophenol/AuNps/APTES/ $\mathrm{Si}(100)$ interfaces as a function of the increasing immersion time in the colloidal solution (5 min-20 min-1 h-24 h). The corresponding SFG, in transmission [20]. However, on silicon in the reflection geometry, the results seem to be very different and counterintuitive. The LSPR does not show up in any positive peak as presented in Fig. 5.

After $5 \mathrm{~min}$, no clear evidence of the LSPR is detected. After $20 \mathrm{~min}$, a single negative feature appears at $525 \mathrm{~nm}$. After $1 \mathrm{~h}$, with a denser AuNps coverage, the dip at $525 \mathrm{~nm}$ is confirmed and a second stronger negative band appears at $660 \mathrm{~nm}$. At $24 \mathrm{~h}$, this latter band dominates the spectrum. Very often, the appearance of shoulder at ca. $650 \mathrm{~nm}$ is indicative of aggregated nanoparticles in a solution. However, in the present case, such a predominant aggregation is confirmed by AFM for $f_{\max }=15 \%$ only. Moreover, these negative features are unusual. All these peculiarities can be explained when studying more closely the analytical expression of the reflectance in the case of AuNps deposited on silicon. The electric field reflected from a surface can be calculated analytically with the Fresnel Formula. In the case of a thin layer on top of the silicon surfaces, the three-layer model already mentioned in SFG/DFG measurements can be used and given that the thin layer is much smaller than the wavelength, the variation of the reflected intensity due to the thin layer is given by:

$\frac{\Delta R}{R}=\frac{8 \pi}{\lambda} \sqrt{\varepsilon_{\mathrm{vac}}} d \operatorname{Im}\left(\frac{\varepsilon_{\mathrm{Si}}-\varepsilon_{\mathrm{MG}}}{\varepsilon_{\mathrm{Si}}-\varepsilon_{\mathrm{vac}}}\right)$.

If divided by $\operatorname{Ln}(10)=2.3$, this formula describes the reflectance spectra actually measured in normal incidence (no distinction anymore between $s$ and $p$ polarization). The input parameters are the complex dielectric functions $\varepsilon=\varepsilon^{\prime}+i \varepsilon^{\prime \prime}$ of the silicon substrate of the surrounding medium (assumed

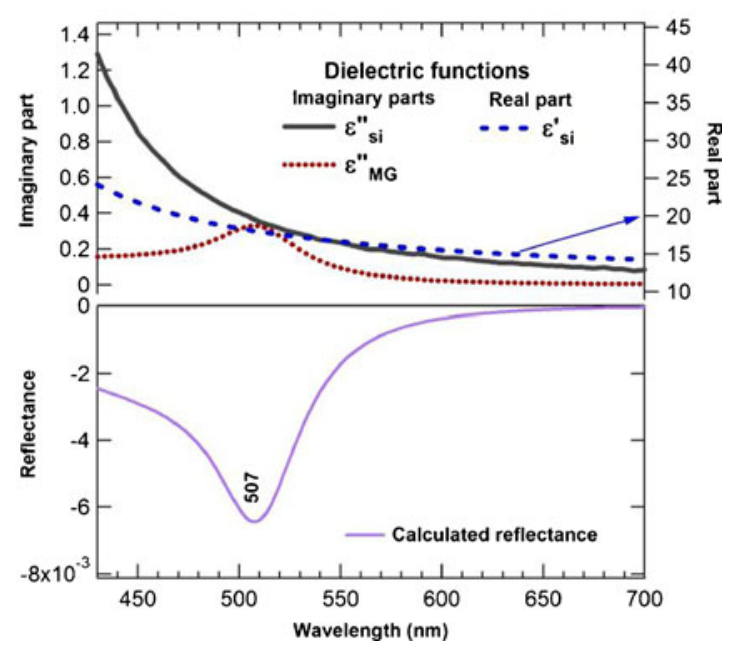

visible and DFG beams wavelengths are given to facilitate comparison between linear and nonlinear optical properties of the samples. Right: calculation of the theoretical reflectance spectrum of AuNps on silicon for $1 \mathrm{~h}$ immersion time within the MG model. See text for explanation 
here to be vacuum for simplification). $\varepsilon_{\mathrm{MG}}$ is the $\mathrm{MG}$ effective medium model approximation of gold spheres in an air matrix as used above. This model satisfactorily captures the plasmon resonance of AuNps (in term of plasmon position and intensity). The analysis of the formula is essential to understand the main features that give the shape to the spectra of Fig. 5 (left panel). With some simplifications, $\varepsilon_{\mathrm{Si}}$ can be considered as mostly real $\left(\varepsilon_{\mathrm{Si}}=18+0.4 \mathrm{i}\right.$ whereas $\left.\varepsilon_{\mathrm{vac}}=1\right)$. As a result, the imaginary part applies to the numerator $\varepsilon_{\mathrm{Si}}-\varepsilon_{\mathrm{MG}}$ only and it simplifies into:

$\left.\frac{\Delta R}{R}\right|_{\text {approx }}=\frac{8 \pi}{\lambda} \sqrt{\varepsilon_{\mathrm{vac}}} d \frac{\varepsilon^{\prime \prime} \mathrm{Si}^{-}-\varepsilon^{\prime \prime} \mathrm{MG}}{17}$.

Therefore, the shape of optical spectrum mostly depends on $\varepsilon_{\mathrm{Si}^{\prime \prime}}{ }^{\prime \prime}-\varepsilon_{\mathrm{MG}}$ " where $\varepsilon$ " is the imaginary part of the dielectric functions. It is extremely instructive to plot the values of $\varepsilon$ "as a function of wavelength as done in Fig. 5 (right panel) in the case of the MG effective medium model approximation. The calculation reproduces the AuNps density corresponding to $1 \mathrm{~h}$ dipping time. The reflectance spectrum will have the shape of the difference between the two curves of the imaginary parts of the dielectric constants of Fig. 5 (right panel), and therefore, it clearly explains the negative appearance of the LSPR peak and is satisfactorily reproduced. Indeed, the calculated spectrum exhibits a negative LSPR peak at $507 \mathrm{~nm}$ instead of $525 \mathrm{~nm}$. This discrepancy is mostly due to the presence of the thiophenol molecules around the AuNps whereas the model considers the AuNps are in vacuum. Moreover, Fig. 5 (right panel) also gives indication about what happens at $660 \mathrm{~nm}$. Since the two plots of the imaginary parts of the dielectric constants $\varepsilon_{\mathrm{Si}^{\prime}}{ }^{\prime \prime}$ and $\varepsilon_{\mathrm{MG}}{ }^{\prime \prime}$ are almost parallel, just a slight tendency for aggregation will display a shallow shoulder in the LSPR peak that will be readily amplified when the difference $\varepsilon_{\mathrm{Si}}{ }^{\prime \prime}-\varepsilon_{\mathrm{MG}}{ }^{\prime \prime}$ is calculated. Therefore, the aggregation peak appears exaggerated in the reflection spectra on silicon.

As a temporary conclusion, the UV-vis spectra of AuNps deposited on silicon confirm the presence of the main plasmon peak at $525 \mathrm{~nm}$ even if it shows up negatively in the reflectance spectra. The negative broad contribution at $660 \mathrm{~nm}$ is due to aggregated AuNps, but it appears strongly exaggerated and does not lead to the conclusion that most of the AuNps are aggregated in agreement with AFM data. Therefore, the amplification of the local field due to the LSPR is still expected at $525 \mathrm{~nm}$ with all these samples.

\section{Discussion}

\section{FTIR versus SFG: molecular sensitivity threshold}

An interesting feature observed by comparison of the FTIR and SFG/DFG measurements is related to the different sensitivity threshold of the molecular fingerprint on small gold nanospheres. We show, in our experimental conditions on the same samples, that SFG/DFG is more sensitive than FTIR. In the latter case (Fig. 3), for $f$ below $9 \%$, no thiophenol is observed. This lack of sensitivity can be explained by the specificity of each technique. In SFG/ $\mathrm{DFG}$, it is a process intrinsically sensitive to the symmetry breaking at the surface of AuNps which produces photons in the visible range easily detected by photomultipliers. Furthermore, the incident visible laser beam is located in the green and exalts the LSPR of the sample (Fig. 5), increasing dramatically the amplitude of the local electric fields around the AuNps and therefore the SFG/ DFG response, which is not the case in FTIR. In this way, we have an absolute measurement of the thiophenol signal, being amplified by the LSPR excitation of the AuNps. Both thiophenol and AuNps constitute the probed interface. Moreover, silicium has no SFG/DFG activity in the involved energies and therefore does not disturb directly the nonlinear process generated by the interface. Nevertheless, we have to remember that the interface reflectivity modulates the nonlinear response through the Fresnel factors contribution. For FTIR, we perform differential measurements with respect to a reference sample without thiophenol. In this way, in the division procedure of the spectra, the sensitivity is related to the IR detector sensitivity. Moreover, we have to compensate for the broad and intense $\mathrm{OH}$ (water molecules) contribution to extract the weak $\mathrm{CH}$ vibration mode of the thiophenol, which becomes tricky for low $f$. The IR sensitivity also depends on surface reflectivity and is well adapted to metal surfaces not for silicon which is dominant for low $\mathrm{N}_{\mathrm{s}}$. As we have no FTIR signal in these conditions, it is more convenient to use the SFG/DFG data for a quantitative analysis of low surfacic molecular coverages. For the present paper, they allow to test the accuracy of the effective medium models developed previously and thus to understand how to take into account interface reflectivity in surfacic spectroscopic tools.

\section{Influence of the interband transition character of gold nanospheres on the SFG/DFG spectra}

By considering the SFG/DFG spectra (Fig. 4) and the parameter $\mathrm{C}_{\mathrm{FG}}$ (Table 1), we see that the baseline intensity of the spectra increases with $\mathrm{N}_{\mathrm{s}}$, with the notable exception of the DFG curve for $24 \mathrm{~h}$ immersion time. This point will be addressed further. Nevertheless, in both cases, it confirms that the contribution of gold nanospheres has a qualitative and quantitative strong impact on the vibrational fingerprint of the spectra. However, the most specific feature of SFG/DFG spectroscopy lies in the interference pattern observed in the spectra. We have the thiophenol vibration mode appearing as a 
dip for SFG and a peak for DFG. It is related to the $\mathrm{s}-\mathrm{d}$ interband electronic transition of gold as mentioned earlier and extensively detailed in previous references $[4,8]$. The experimental marker of this electronic effect is the phase shift $\phi_{\mathrm{FG}}$. It is the strongest when $\phi_{\mathrm{FG}}$ equals $90^{\circ}$. In the present work, the SFG wavelength coincides with the maximum of the s-d interband transition located in the blue $\left(460 \mathrm{~nm} ; \phi_{\mathrm{FG}} \sim\right.$ $90^{\circ}$ ); therefore, the gold contribution to the nonlinear response is stronger than in the DFG case, whose wavelength is in the red $\left(640 \mathrm{~nm} ; \phi_{\mathrm{FG}} \sim 20^{\circ}\right)$. This resonance effect of the SFG beam with the $s-d$ interband electronic transition explains why we have a destructive interference pattern strongly marked in SFG and a constructive one in DFG. Moreover, it explains a big difference with a previous study [8] where $\phi_{\mathrm{FG}}$ was close to $140^{\circ}$ for $\mathrm{SFG}$ and $70^{\circ}$ for DFG. This is because the SFG and DFG wavelengths were at 500 and $550 \mathrm{~nm}$ (IR spectral range centred on $10 \mu \mathrm{m}$ ), i.e. well beyond the maximum of the s-d interband transition. In summary, in our SFG/ DFG data, due to the considered wavelengths, the SFG spectra are strongly influenced by the interband electronic transition of gold while DFG is only weakly affected. This property is very interesting because it proves that the vibration detected at $3,057 \mathrm{~cm}^{-1}$ is due to thiophenol molecules which are adsorbed on gold. In the case these molecules would have been adsorbed on other substrates, the interference pattern would have been different.

\section{Influence of the reflectivity of gold nanospheres on the SFG/DFG spectra}

As calculated in "SFG/DFG measurements" for SFG/DFG and observed in "UV-vis measurements" for UV-vis, the surface coverage of AuNps $\left(\mathrm{N}_{\mathrm{s}}\right)$ plays a crucial role on the optical response of the sample. Depending on the dipping time in the AuNps solution, the molecular amplitude $\mathrm{a}_{z z z}$ is expected to scale linearly with $\mathrm{N}_{\mathrm{s}}[8,21]$ because our samples produce SFG/DFG photons in a coherent way (cf. "Nonlinear optical vibrational spectroscopy"). To illustrate the influence of the sample reflectivity, we report the evolution of $\mathrm{a}_{\mathrm{zzz}}$ in Fig. 6. In this manner, it is possible to compare and discuss the accuracy or discrepancy between the molecular amplitudes within the framework of the effective medium models developed above. The error bars are related to the measurement uncertainty of $\mathrm{N}_{\mathrm{s}}$ and $f$ extracted by AFM (Tables 1 and 2).

In spite of the small number of points per curve, we checked the correctness of the linear fit hypothesis by performing power fits of $\mathrm{a}_{\mathrm{zzz}}$ as a function $\mathrm{N}_{\mathrm{s}}$ with a free exponent $n$. The mean values for $n$ are 1.57 for SFG and 1.31 for DFG. In addition, this analysis shows that linearity in the $\mathrm{BM}$ model is better than in the MG frame, as the $n$ values are closer to 1 (1.49 and 1.24 for SFG and DFG, respectively). However, these two effective medium models are not sufficient to fully account for the linear hypothesis as shown by the slight deviations from the ideal case. A quick inspection of Fig. 5 (left) shows that, from the energy point of view, DFG is not influenced in the same way as SFG. The former coincides with the broad band in the red related to AuNps aggregation (ca. 650-660 nm) at high $\mathrm{N}_{\mathrm{s}}(1-24 \mathrm{~h}$ dipping time). The latter does not undergo any local electronic effect, but the $\mathrm{s}-\mathrm{d}$ interband transition as explained before. At low $\mathrm{N}_{\mathrm{s}}(5-$ 20 min dipping time), it should be noted that only the LSPR of isolated AuNps may impact the reflectivity through its excitation by the incident visible beam in the nonlinear processes. For both cases, it proves that the LSPR contribution is not correctly accounted for by MG nor BM as shown in "UVvis measurements" where the LSPR is calculated at $507 \mathrm{~nm}$ instead of the expected value of $525 \mathrm{~nm}$. It is a clear indication that the local fields at the visible SFG and DFG wavelengths play a significant role. The fact that the vibration amplitude linearly increases with $\mathrm{N}_{\mathrm{s}}$ for both SFG and DFG is also consistent with the previous observation of an exaltation factor for the detection of molecules adsorbed on AuNps. Indeed,
Fig. 6 Evolution of the molecular amplitude $\mathrm{a}_{\mathrm{zzz}}$ as a function of the surface coverage of AuNps on silicon $\left(\mathrm{N}_{\mathrm{s}}\right)$ for $\mathrm{SFG}$ (left) and DFG (right) within the Maxwell-Garnett and Bruggeman effective medium models. Error bars depend on the precision of $\mathrm{N}_{\mathrm{s}}$ deduced by AFM measurements (Table 1). The dashed curves are the best linear fits to data

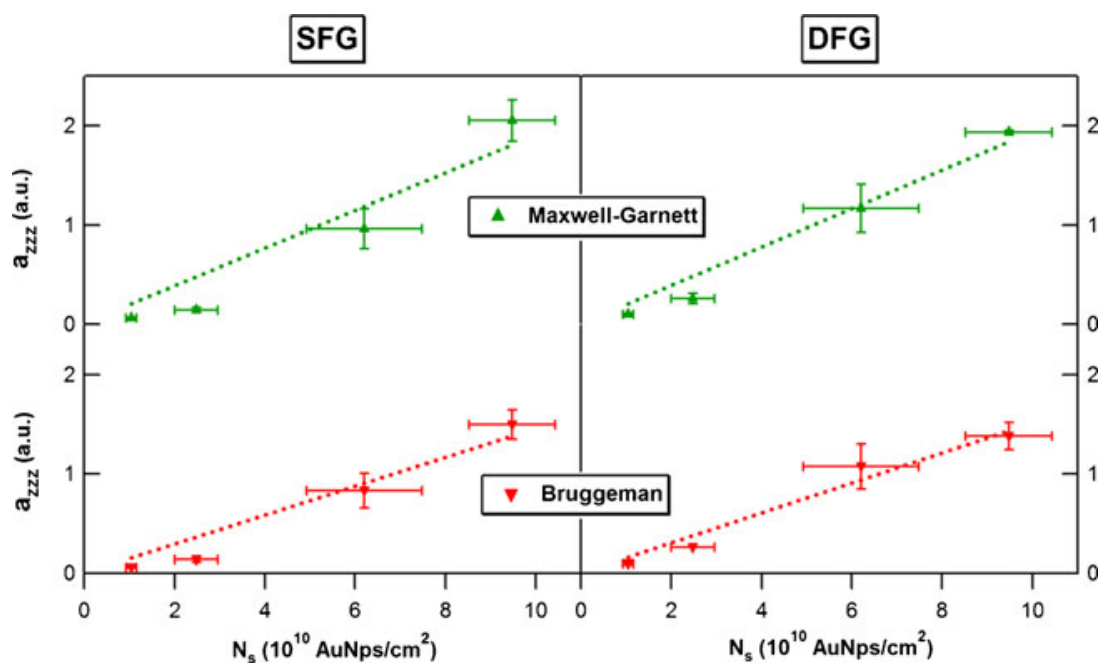


we had previously estimated this factor to be around 20 to explain the differences observed with a flat gold surface covered by a full monolayer of thiophenol [4]. However, this latter result implied that the vibrational amplitude increased linearly with $\mathrm{N}_{\mathrm{s}}$, which is confirmed in the present work.

\section{Conclusions}

In summary, we have studied and compared the submonolayer sensitivity threshold of thiophenol adsorbed on AuNps as small as $17 \mathrm{~nm}$ and grafted on silicon for different filling factors $f$ checked by AFM imaging. We showed that, on silicon, $\mathrm{SFG} / \mathrm{DFG}$ spectroscopy is intrinsically more sensitive than conventional FTIR spectroscopy especially for $f$ below $9 \%$. Moreover, we have shown that SFG/DFG spectroscopy could take profit of the LSPR amplification by the incident visible laser beam to enhance the sensitivity without the need of hot spots as mandatory required for instance in SERS spectroscopy of small nanoparticles with similar diameters. We have quantitatively related the nonlinear SFG/DFG molecular amplitudes to the samples reflectance measured by UV-vis measurements by developing the three-layer effective medium models of $\mathrm{MG}$ and $\mathrm{BM}$ and showing that the $\mathrm{BM}$ approach was more appropriate to explain the SFG and DFG data when AuNps aggregation occurs. As a consequence, our results show that no coupling of nonlinear optical spectroscopy with the optical properties of aggregated AuNps occurs. For small AuNps, only the LSPR amplification of isolated AuNps can be coupled to nonlinear optics to boost the molecular sensitivity for $f$ ranging from 1 to $4 \%$. Finally, we illustrated that the effective medium models of MG and BM were not sufficient to precisely take into account the LSPR coupling with the adsorbed molecules. Neglecting the molecular contribution was not possible to properly quantify the coupling between plasmonics and nonlinear optical vibrational spectroscopy. To go further quantitatively and describe the role of the local fields, the next steps should include a continuous tuning of the incident visible laser beam on several samples of different AuNps densities. In this perspective, the fine tuning of the LSPR with the visible, SFG or DFG beams would allow to increase the molecular sensitivity required in plasmonic plateforms used as biosensors where the precise targeting of specific chemical bonds between molecular probes and targets is of crucial importance to characterize the biomolecular recognition process at play in those systems.

\footnotetext{
Acknowledgments Research leading to these results has been supported by the Région Île-de-France in the framework of the funding program C'Nano IdF under grant agreement CREMOSOFT. The authors acknowledge the BQR financial support of the Université Paris-Sud. The authors also acknowledge C. Six and A. Gayral for technical assistance on the SFG/DFG experimental setup and L. Dalstein for fruitful discussion on theoretical aspects of SFG and SFS spectroscopy of nanoparticles.
}

Open Access This article is distributed under the terms of the Creative Commons Attribution License which permits any use, distribution, and reproduction in any medium, provided the original author(s) and the source are credited.

\section{References}

1. Morel AL, Boujday S, Méthivier C, Krafft JM, Pradier CM (2011) Biosensors elaborated on gold nanoparticles: a PMIRRAS characterisation of the IgG binding efficiency. Talanta $85: 35-42$

2. Barbillon G, Bijeon JL, Plain J, de la Chapelle ML, Adam PM, Royer P (2007) Electron beam lithography designed chemical nanosensors based on localized surface plasmon resonance. Surf Sci 601:50575061

3. Enders D, Nagao T, Pucci A, Nakayam T (2006) Reversible adsorption of Au nanoparticles on $\mathrm{SiO}_{2} / \mathrm{Si}$ : an in situ ATR-IR study. Surf Sci 600:L71-L75

4. Pluchery O, Humbert C, Valamanesh M, Lacaze E, Busson B (2009) Enhanced detection of thiophenol adsorbed on gold nanoparticles by SFG and DFG nonlinear optical spectroscopy. Phys Chem Chem Phys 11:7729-7737

5. Tourillon G, Dreesen L, Volcke C, Sartenaer Y, Thiry PA, Peremans A (2007) Total internal reflection sum-frequency generation and dense gold nanoparticles monolayer: a route for probing adsorbed molecules. Nanotechnology 18:415301, 1-7

6. Humbert C, Busson B, Abid JP, Six C, Girault HH, Tadjeddine A (2005) Self-assembled organic monolayers on gold nanoparticles: a study by sum-frequency generation combined with UV-vis spectroscopy. Electrochim Acta 50:3101-3110

7. Bordenyuk AN, Weereman C, Yatawara A, Jayathilake HD, Stiopkin I, Liu Y, Benderskii AV (2007) Vibrational sum frequency generation spectroscopy of dodecanethiol on metal nanoparticles. J Phys Chem C 111:8925-8933

8. Humbert C, Pluchery O, Lacaze E, Tadjeddine A, Busson B (2012) A multiscale description of molecular adsorption on gold nanoparticles by nonlinear optical spectroscopy. Phys Chem Chem Phys 14:280 289

9. Dreesen L, Sartenaer Y, Humbert C, Mani AA, Méthivier C, Pradier CM, Thiry PA, Peremans A (2004) Probing ligand-protein recognition with sum-frequency generation spectroscopy: the avidinbiocytin case. Chem Phys Chem 5:1719-1725

10. Sartenaer Y, Tourillon G, Dreesen L, Lis D, Mani AA, Thiry PA, Peremans A (2007) Sum-frequency generation spectroscopy of DNA monolayers. Biosens Bioelectron 22:2179-2183

11. Walter SR, Geiger FM (2010) DNA on stage: showcasing oligonucleotides at surfaces and interfaces with second harmonic and vibrational sum frequency generation. J Phys Chem Lett 1:9-15

12. Howell C, Zhao J, Koelsh P, Zharnikov M (2011) Hybridization in ssDNA films: a multi-technique spectroscopy study. Phys Chem Chem Phys 13:15512-15522

13. Tourillon G, Dreesen L, Volcke C, Sartenaer Y, Thiry PA, Peremans A (2009) Close-packed array of gold nanoparticles and sum frequency generation spectroscopy in total internal reflection: a platform for studying biomolecules and biosensors. J Mater Sci 44:6805-6810

14. Humbert C, Busson B (2011) SFG spectroscopy of biointerfaces. In: Pradier CM, Chabal YJ (eds) Biointerface characterization by advanced IR spectroscopy. Elsevier, Amsterdam, pp 279-321

15. Ji XH, Song XN, Li J, Bai YB, Yang WS, Peng XG (2007) Size control of gold nanocrystals in citrate reduction: the third role of citrate. J Am Chem Soc 129:13939-13948 
16. Roke S, Gonella G (2012) Nonlinear light scattering and spectroscopy of particles and droplets in liquids. Annu Rev Phys Chem 63:353378

17. de Aguiar HB, Scheu R, Jena KC, de Beer AGF, Roke S (2012) Comparison of scattering and reflection SFG: a question of phase-matching. Phys Chem Chem Phys 14:68266832

18. Feugmo CGT, Liégeois V (2013) Analyzing the vibrational signatures of thiophenol adsorbed on small gold clusters by DFT calculations. ChemPhysChem 14:1633-1645
19. Aspnes DE, Theeten JB (1979) Investigation of effective-medium models of microscopic surface roughness by spectroscopic ellipsometry. Phys Rev B 20:3292-3302

20. Pluchery O, Lacaze E, Simion M, Miu M, Bragaru A, Radoi A (2010) Optical characterization of supported gold nanoparticles for plasmonic biosensors. Semiconductor Conference (CAS), International Sinaia, IEEE Electron Devices Society, Romania, pp 159-162

21. Zhuang X, Miranda PB, Kim D, Shen YR (1999) Mapping molecular orientation and conformation at interfaces by surface nonlinear optics. Phys Rev B 59:12632-12640 